\title{
Posterior Reversible Encephalopathy Syndrome Mimicking a Left Middle Cerebral Artery Stroke
}

\author{
Santo Terranova, Jai Dev Kumar and Richard B. Libman*
}

Department of Neurology, Long Island Jewish Medical Center, New Hyde Park, NY, USA

\begin{abstract}
Certain Acute Clinical presentations are highly suggestive of stroke caused by specific mechanisms. One example of this would be the sudden onset of aphasia without hemiparesis often reflecting cerebral embolism, frequently from a cardiac source. Posterior reversible encephalopathy syndrome (PRES) describes a usually reversible neurologic syndrome with a variety of presenting symptoms from headache, altered mental status, seizures, vomiting, diminished spontaneity and speech, abnormalities of visual perception and visual loss. We report a patient presenting with elevated blood pressure, CT characteristics of PRES but a highly circumscribed neurologic syndrome (Wernicke's Aphasia without hemiparesis) suggestive of a cardioembolic stroke affecting the left MCA territory. That is, PRES mimicked a focal stroke syndrome. The importance of recognizing this possibility is that his deficits resolved with blood pressure control, while other treatments, such as intensifying his anticoagulation would have been inappropriate. In addition, allowing his blood pressure to remain elevated as is often done in the setting of an acute stroke might have perpetuated the underlying pathophysiology of PRES leading to a worse clinical outcome. For this reason PRES needs to be recognized quickly and treated appropriately.
\end{abstract}

Keywords: Posterior reversible encephalopathy syndrome, Stroke, Embolism, Wernicke's aphasia.

\section{INTRODUCTION}

The syndrome of sudden aphasia without hemipresis often suggests acute embolic stroke. Our aim in this case report is to remind clinicians that other conditions may produce a similar clinical syndrome but have a very different therapeutic implications. incoherent, not making sense, or confused. Admission blood pressure was 212/104; he was afebrile, Neurologic exam showed severe Wernicke's aphasia and a right visual field deficit. The remainder of the neurologic exam was normal. He had a history of atrial fibrillation, ischemic cardiomyopathy, hypertension, dyslipidemia, and internal defibrillator. Medications included warfarin. The patient was clinically

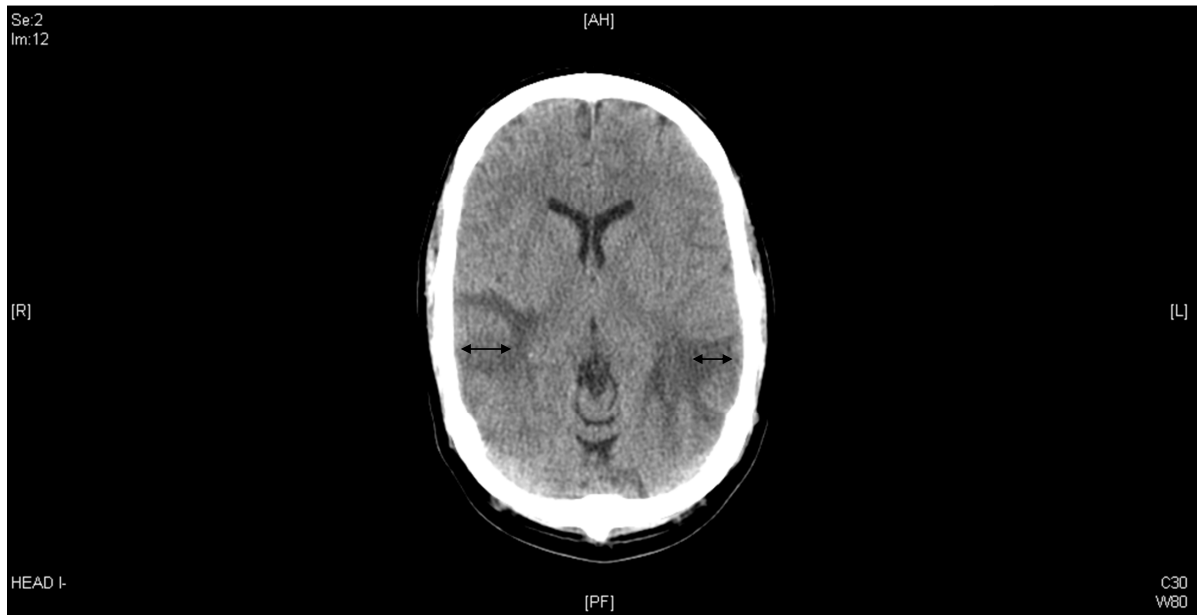

Fig. (1). CT scan showed subcortical white matter hypodensity with slight mass effect in bilateral posterior frontal, parietal, occipital, and temporal lobes most consistent with vasogenic edema (arrows).

A 54 year old left handed white gentleman was evaluated at our institution for acute aphasia. He had developed an acute speech disturbance, described by observers as being

*Address correspondence to this author at the Division of Neurology, Harvey Cushing Institutes of Neuroscience, North Shore-LIJ Health System, USA; Tel: (718) 470-4490; Fax: (718) 347-3016; E-mail: rlibman@lij.edu diagnosed with a left middle cerebral artery (MCA) infarction likely secondary to cardiac embolism given his history. Initial Head CT without contrast done in slightly under 6 hours from presentation showed no evidence of acute left middle cerebral infarct or hemorrhage but showed subcortical white matter hypodensity with slight mass effect in bilateral posterior frontal, parietal, occipital, and temporal lobes most consistent with vasogenic edema. The edema pat- 
tern was bilateral and fairly symmetric (Fig. 1). MRI could not be done due to his pacemaker. His blood pressure was subsequently brought down to $128 / 72$ over a 24 hour period at which point there was significant improvement of his symptoms. Repeat CT Head with contrast done the following day showed diffuse white matter low attenuation with sulcal effacement involving, the splenium of the corpus callosum, without abnormal enhancement (Fig. 2). His aphasia continued to improve over several days at which time his speech

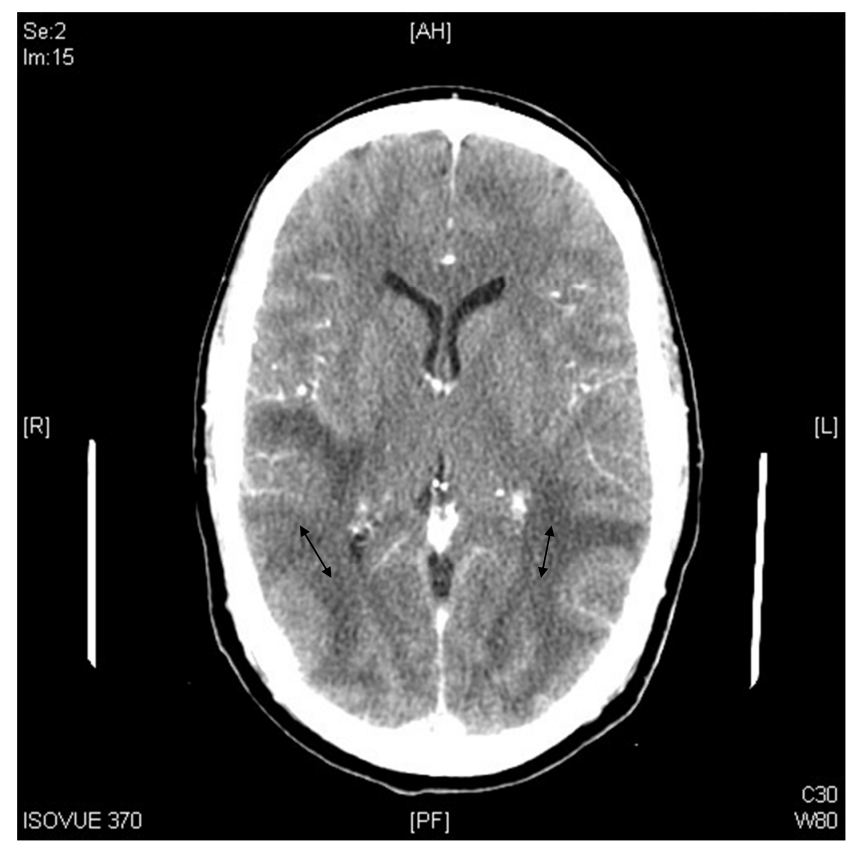

Fig. (2). CT Head with contrast done the following day showed diffuse white matter low attenuation with sulcal effacement, involving the splenium of the corpus callosum, without abnormal enhancement.

showed only very mild circumlocutions and word finding difficulty. The right visual field deficit resolved. His mild neurologic deficits continued to improve and had resolved completely at two month follow up. Repeat CT head with and without contrast done four months after presentation showed that the symmetric white matter hypodensities had completely resolved. There was no evidence of enhancing masses or acute infarcts (Fig. 3).

Posterior Reversible Encephalopathy Syndrome (PRES) was first described in 1996, presenting with headache, seizures, changes in mental status, and various visual deficits [1]. It has been characterized on brain imaging as symmetric parieto-occipital edema but can occur with varying imaging appearances. It describes a usually reversible neurologic syndrome with a variety of presenting symptoms from headache, altered mental status, seizures, vomiting, diminished spontaneity and speech and abnormalities of visual perception and visual loss. Causes of PRES include hypertension, eclampsia, immunosuppressive medications, hypercalcemia, thrombocytopenic syndromes, amyloid angiopathy, systemic lupus erythematosus and renal failure. The pathophysiology of PRES is related to a hyperperfusion state whereby elevated capillary hydrostatic pressure leads to autoregulation dysfunction. There is blood brain barrier breakdown and extravasation of fluid resulting in edema. In addition to these primary processes secondary events may occur which include focal cerebral vasospasm or thrombosis leading to brain infarction and hemorrhage. The most frequent MRI findings associated with PRES are hyperintensities on fluid-attenuated inversion recovery (FLAIR) images in the parietooccipital and posterior frontal cortical and subcortical white matter. Less commonly, the brain stem, basal ganglia, and cerebellum are involved. Atypical imaging appearances would include contrast enhancement, hemorrhage and restricted diffusion on MRI [2].

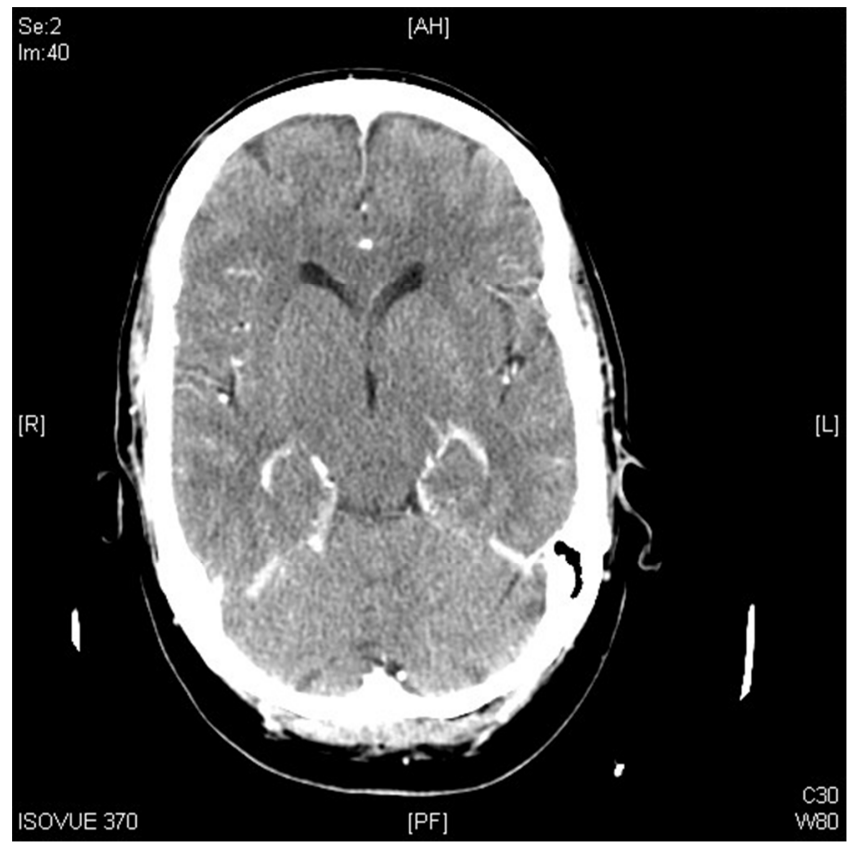

Fig. (3). CT head with contrast done four months after presentation showed that the symmetric white matter hypodensities had completely resolved.

It is important to note that the calcarine and paramedian occipital lobe structures are usually spared, a fact that distinguishes PRES from bilateral infarction of the Posterior cerebral artery distribution [1]. The reason that PRES tends to favor the posterior circulation is not clear. However, it may be due to a lack of sympathetic innervation at the level of the arterioles supplied by the vertebrobasilar system compared with the anterior circulation [2].

Our patient presented with elevated blood pressure, CT characteristics of PRES but a highly circumscribed neurologic syndrome (Wernicke's aphasia without hemiparesis) suggestive of a cardioembolic stroke affecting the left MCA territory. That is, PRES mimicked a focal stroke syndrome. The importance of recognizing this possibility is that his deficits resolved with blood pressure control, while other treatments, such as intensifying his anticoagulation would have been inappropriate. In addition, allowing his blood pressure to remain elevated as is often done in the setting of an acute stroke might have perpetuated the underlying pathophysiology of PRES leading to a worse clinical outcome. It is for this reason that PRES needs to be recognized quickly and treated appropriately. 


\section{REFERENCES}

[1] Hinchey J, Chaves C, Appignani B, et al. A reversible posterior leukoencephalopathy syndrome. N Engl J Med 1996; 334: 494-500.
[2] McKinney AM, Short J, Truwit CL, et al. Posterior reversible encephalopathy syndrome: incidence of atypical regions of involvement and imaging findings. Am J Roentgenol 2007; 189: 904-12.

Received: June 24, 2011

Revised: September 29, 2011

Accepted: November 07, 2011

(C) Terranova et al.; Licensee Bentham Open.

This is an open access article licensed under the terms of the Creative Commons Attribution Non-Commercial License (http://creativecommons.org/licenses/by$\mathrm{nc} / 3.0 /$ ), which permits unrestricted, non-commercial use, distribution and reproduction in any medium, provided the work is properly cited. 